\title{
The demand for neonatal intensive care
}

\author{
D J Field, S Hodges, E Mason, P Burton, J Yates, S Wale
}

\begin{abstract}
In a one year prospective study within the Trent Regional Health Authority the demand for neonatal intensive care was estimated to be $1 \cdot 1$ cots per 1000 births. Intensive care level 1 (as defined by the British Paediatric Association and British Association for Perinatal Paediatrics) was determined by two separate techniques, which showed close agreement. Intensive care level 2 could not be measured directly, as the definition was too subjective. This aspect of demand was therefore estimated by using data derived from the treatment of babies transferred for intensive care.

These findings represent a minimum estimate of need, as the data were obtained from a service constrained by having facilities well below the estimated level (roughly $60 \%$ of estimated demand). In the future other factors such as increased survival of extremely preterm infants will be likely to increase demand still further.
\end{abstract}

\section{Introduction}

In September 1988 the Royal College of Physicians of London recommended that neonatal services should be increased from the current level of less than 1 intensive care cot per 1000 births to $1 \cdot 5$ cots per 1000 . Criticism of the report has centred on the lack of direct evidence to support the recommended increase. ${ }^{2}$ In particular, no clear evidence was given by the royal college committee for the suggested increase in provision compared with the previous recommendations of 0.5 cot per 1000 births made in 1971 and 1.0 cot per 1000 made in $1977 .{ }^{3+}$ The lack of objective data both on the demand for intensive care and to a lesser extent the supply has seriously hampered the planning of neonatal care services in the past 20 years.

Among those concerned in neonatal care there is strong feeling that the current provision of intensive care cots is inadequate, and in some areas the demand regularly exceeds supply. As a result in parts of Britain clinicians experience considerable difficulty in finding available intensive care cots.

Trent is the second largest regional health authority in the United Kingdom. The population is 4.6 million with around 55000 births occurring annually. There are 17 consultant obstetric units with associated infant care facilities. All these units are capable of special care but have a variable capacity in terms of intensive care. There are three teaching hospitals, three units with 5000 or more deliveries a year, and five units in which neonatal intensive care is regularly undertaken. The region was known to be virtually self sufficient for neonatal intensive care and was therefore highly suited to a study of neonatal intensive care in terms of supply and demand.

\section{Methods}

A one year prospective study of the use of intensive care was undertaken within the Trent health region from February 1987 to January 1988 inclusive. All 17 units participated.

Neonatal intensive care is normally subdivided into three main categories as defined by the British Paediatric Association and British Association for Perinatal Paediatrics. ${ }^{6}$ These are intensive care level 1 , intensive care level 2, and special care (see appendix). We wished to estimate the combined workload of levels 1 and 2. The appendix shows that level 1 includes babies receiving specific treatments (artificial respiratory support or intravenous nutrition), and this type of care is easy to identify. For level 2 care and special care several specific criteria are listed, but the main group numerically in level 2 care is described as "unstable infants" and an important group in the special care definition is "babies requiring continuous monitoring." Clearly the distinction between these two groups is open to interpretation, but it is important to differentiate between these two aspects of care in assessing the demand for intensive care. It was our impression that most level 2 workload arose from infants admitted for level 1 care but who subsequently required a period of level 2 care before entering the special care category. These changes in state are not normally marked by specific events except for infants transferred to a centre for intensive care. In this setting lack of intensive care beds, policy of referral centres, and social considerations ensure transfer back to the hospital of origin once the baby reaches the special care category.

Thus in infants transferred to a centre for intensive care that admission represents days of level 1 care plus days of level 2 care. As days of intensive care level 1 and total length of stay may reliably be obtained, we decided to use this information to derive a ratio of level 1 days to level 2 days for transferred infants. Infants who did not complete a successful return to the hospital of origin, either because the infant died or because the parents did not wish to have the infant transferred back, were not included in calculating the ratio. The method ignores level 2 days produced by infants not entering the level 1 category-for example, infants requiring an exchange transfusion; numerically these were considered unimportant.

Level 1 days and total patient days (level 1, level 2, and special care) were calculated in two ways. Firstly, senior medical and nursing staff within each unit recorded the workload daily at midday-that is, the total number of inpatients and number of babies receiving level 1 care. (For comparison infants considered to be receiving level 2 care were also noted.) This point estimate of care was used to represent the workload for that day. Secondly, one of four independent observers (two doctors, two nurses) visited each unit at frequent intervals and recorded the type and duration of treatment given to every infant admitted during the year of the study. This approach permitted level 1 care and total length of stay to be recorded but not level 2 care, which was too subjective to be assessed in this way.

Both methods of data collection provided data for a 
complete 12 month period, but these were not identical. Staff within the units collected data on inpatients during the specific 12 months of the study irrespective of the dates of admission and discharge. The observers collected data relating to the whole stay of each baby whose admission date fell during the year of the study irrespective of discharge date. It was thought that this would result in only minor differences of calculated demand.

In order to calculate cot requirements a $70 \%$ level of occupancy was assumed, most other investigations having used this figure.'

\section{Results}

The table gives the results relating to intensive care level 1. Results are expressed as the total numbers of days of intensive care level 1 as recorded by individual units and by independent observers. The data collected by independent observers were calculated from 852 separate admissions. In general the two sets of data showed good agreement, but some large percentage differences were present in the smaller units. Small variations in the total for each unit may have been caused either by the slightly different study populations or by a rounding up bias by observers. In an attempt to reduce the rounding up bias 0.5 day of intensive care was subtracted from the individual total for each patient in the relevant column of the table.

Total days of intensive care level 1 and total bed occupancy in the various hospital units

\begin{tabular}{|c|c|c|c|c|}
\hline \multirow[b]{2}{*}{ Unit } & \multicolumn{2}{|c|}{ Days of level 1 care } & \multicolumn{2}{|c|}{$\begin{array}{c}\text { Total bed occupancy (days): } \\
\text { all levels of care }\end{array}$} \\
\hline & $\begin{array}{l}\text { Data collected } \\
\text { by each unit }\end{array}$ & $\begin{array}{c}\text { Data on } \\
\text { individual } \\
\text { babies } \\
\text { collected by } \\
\text { independent } \\
\text { observer }\end{array}$ & $\begin{array}{c}\text { Data collected } \\
\text { by each unit }\end{array}$ & $\begin{array}{c}\text { Data on } \\
\text { individual } \\
\text { babies } \\
\text { collected by } \\
\text { independent } \\
\text { observer }\end{array}$ \\
\hline 1 & 302 & 139 & 2020 & 1771 \\
\hline 2 & 119 & 119 & 2954 & 2599 \\
\hline 3 & 416 & 420 & 3621 & 4077 \\
\hline 4 & 1518 & 1200 & 5965 & 6027 \\
\hline 5 & 4 & 5 & 1973 & 1786 \\
\hline 6 & 33 & 25 & 3117 & 3027 \\
\hline 7 & 102 & 107 & 2752 & 2630 \\
\hline 8 & 69 & 45 & 1538 & 1564 \\
\hline 9 & 18 & 68 & 3159 & 3352 \\
\hline 10 & 438 & 583 & 4640 & 4967 \\
\hline 11 & 1643 & 1590 & 6800 & 6943 \\
\hline 12 & 657 & 605 & 4701 & 4843 \\
\hline 13 & 7 & 44 & 4028 & 3785 \\
\hline 14 & 25 & 10 & 1165 & 1272 \\
\hline 15 & 15 & 43 & 1825 & 1805 \\
\hline 16 & 1168 & 1217 & 6468 & 6480 \\
\hline 17 & 77 & 116 & 3448 & 3326 \\
\hline Total (days) & 6611 & 6336 & 60174 & 60254 \\
\hline
\end{tabular}

Larger numerical differences seen only in units 1 and 4 probably represented a failure of staff within the units to adhere to definitions of intensive care. Nevertheless, the two regional totals differed by only $4 \%$ ( 275 days).

The table also gives the figures for total cot days (levels 1 and 2 and special care days combined) for each unit, collected both within the individual units and by independent observers. There was a close relation between the two sets of data, variation in the totals for each unit probably resulting from minor differences in the study population. The two totals for the region as a whole differed by only $0 \cdot 1 \%$ ( 80 days).

The ratio for estimating level 2 intensive care was calculated after analysis of 162 "round trip" transfers - that is, transfer to a referral centre and back to the hospital of origin. Data collected by observers indicated that the mean duration of level 1 care for these infants at the referral unit was $6 \cdot 14$ days with, on average, a further $8 \cdot 41$ days before transfer back. This second figure was taken to represent level 2 days for these infants and implied a simple ratio of level 1 to level 2 of $1: 1 \cdot 37$ days.

The distributions of the duration of intensive care level 1 and intensive care level 2 both showed a pronounced skew to the right and a roughly constant coefficient of variation. Both distributions included several zero values. Such distributions may prove difficult to analyse and it was recognised that the crude ratio of means for level 1 and level 2 might therefore prove to be misleading. The use of a simple logarithmic transformation ( $\log _{\mathrm{e}}$ ("days")) was impossible because of the zero values, and a $\log _{\mathrm{e}}$ ("days" $+\mathrm{k}$ ) transformation led to analytical instability and results which were highly dependent on the particular value of $\mathrm{k}$ added. An alternative approach to analysis was therefore taken. The variable "days" was transformed by adding the very small increment 0.01 and the transformed variable then modelled as a gamma distribution ${ }^{7}$ by using a logarithmic link in GLIM (generalised linear interactive modelling) $3 \cdot 77 .{ }^{8}$ This produced a stable, well fitting model. With this technique the estimated ratio between level 1 and level 2 was found to be 1.37 ( $95 \%$ confidence interval 1.04 to 1.80 ), which was identical with the simple estimate and suggested that the original use of 1.37 was acceptable. Furthermore, the estimate of the ratio was similar for the three main hospitals that treated most of the infants transferred $(1.36,1.36,1.20)$. The estimated ratio in the fourth hospital accepting transfers was higher $(1 \cdot 64)$, but this was based on only six cases. The result of a formal statistical test for a difference among the four centres was not significant $(p>0 \cdot 75)$.

The transferred infants seemed typical of the overall population who required intensive care level 1. By using the same modelling technique as above the distribution of intensive care days was shown to be similar for the transferred and non-transferred survivors (ratio $1 \cdot 11$ (95\% confidence interval 0.85 to $1 \cdot 46) ; \mathrm{p}=0 \cdot 44)$.

Total days of level 2 care were therefore derived for all units by multiplying the figures in the table (data from independent observers on days of level 1 care) by $1 \cdot 37$. The total number of level 2 days for the region calculated in this way was $8680(6336 \times 1 \cdot 37)$.

By using the British Paediatric Association and British Association for Perinatal Paediatrics definition the units themselves identified a lower figure $(4458$ days). Within the unit data we noted no relation between days of level 1 care and days of level 2 care. Even for the three main hospitals with large numbers of infants the ratio of recorded level 1 to recorded level 2 care showed great variation $(1 \cdot 05,0 \cdot 19,0 \cdot 30)$. This variation was not present in other important aspects of their work (duration of ventilation, overall length of stay) and confirmed our view that using the definitions alone was unsuitable for assessing level 2 care. The formula produced a higher figure for level 2 days in 10 units, a lower figure in six, and was identical in one.

A total of 55692 births occurred in participating units during the year of the study. Total births for the Trent region in 1986 (latest figures available) were 55750. The first figure was therefore considered appropriate for calculating the demand for intensive care in relation to births.

Total days of neonatal intensive care were derived by adding days of level 1 intensive care collected by independent observers to calculated days of level 2 care -15016 cot days or $41 \cdot 1$ cots per day with $100 \%$ occupancy. With the assumption of $70 \%$ occupancy this represents 59 intensive care cots within the region or $1 \cdot 1$ per 1000 births.

These figures do not include work generated by 14 premature infants transferred out of the region for intensive care (estimated to represent a further 200 days of intensive care) and intensive care of 
some infants treated surgically when it occurred in a paediatric as opposed to a neonatal unit (estimated to represent a further 180 days of intensive care). The figures do include 490 days of intensive care generated by babies from outside the region. The combined effect of these three factors did not alter the estimate of $1 \cdot 1$ cots per 1000 births.

\section{Discussion}

This study was aimed at identifying the demand for neonatal intensive care in the Trent region, which was shown to be $1 \cdot 1$ intensive care cots per 1000 births. The reliability of this information was confirmed by using two methods of data collection. By using this approach it was possible to verify two aspects of the data. These data were intensive care level 1 days and total length of stay (total bed days).

The figures relating to intensive care level 2 were less clear cut. In view of the subjective nature of the data when based entirely on interpretation of the definitions of the British Paediatric Association and British Association for Perinatal Paediatrics it seemed essential to apply an alternative approach to this aspect of neonatal care. A calculation based on the suitability of transferred infants for discharge from intensive care seemed to offer an adequate practical alternative. This approach has the particular attraction of estimating the need for intensive care level 2 in a group of babies whose state is likely to be the subject of regular consideration, unlike infants whose entire course is spent within one unit and whose transition to true special care is rarely signified by a specific event. For most infants we think that this method is a fair assessment of demand for level 2 care. This aspect of the study will need confirmation in an investigation designed to look specifically at this point.

Similar published work is limited. The study which has provided the most reliable guide to demand for neonatal intensive care was a retrospective investigation carried out in the North East Thames health region. ${ }^{9}$ That study estimated the requirement for intensive care by reviewing the case notes of infants admitted for intensive care. The data were then extrapolated to provide a formula capable of predicting demand for neonatal intensive care based on the number of infants in each weight group. The study could not separate intensive care level 1 from intensive care level 2 and had the additional problem that the end of intensive care had to be judged retrospectively. In addition, only a subgroup of 82 infants were assessed in this way.

In the past most recommendations from learned bodies regarding the provision of neonatal intensive care facilities have been based on limited data. Recent examples have ranged between 1.3 and 1.5 intensive care cots per 1000 births. ${ }^{10} 11$ Our data suggest that the day to day demand in the Trent region is at present below this level. The results of our analysis are influenced by the particular ratio (1.37) that was estimated between the number of days at intensive care level 1 and the number at level 2 . Given that this figure is subject to statistical variation, the estimated use of intensive care cots was recalculated by using ratios 1.04 and 1.80 (the $95 \%$ confidence interval for the ratio). These ratios gave answers of 0.9 and 1.25 per 1000 births and it is therefore likely that the true utilisation of neonatal intensive care cots in the Trent region lies somewhere between these two figures. These results, however, should be considered to provide a minimum estimate of the real demand for intensive care, as the study, which was based on utilisation, was carried out in a region whose actual neonatal intensive care provision is restricted, and utilisation will inevitably underestimate true demand.
It is difficult to describe the present situation regarding neonatal care facilities in a manner acceptable to all interested parties. Neonatal physicians in general define provision in terms of the guidelines of the British Paediatric Association and British Association for Perinatal Paediatrics, whereas neonatal nurses tend to focus more specifically on nursing numbers and levels of equipment. By contrast, management views provision in terms of the original capital investment and the number of cots provided at that stage. These approaches do not agree, but by each of these criteria there was a definite shortfall. Even the management view would identify a shortage of 22 (37\%) cots compared with demand.

There are likely to be pronounced regional variations in demand for neonatal intensive care, and for some health authorities 1.5 intensive care cots per 1000 births may be the appropriate level. Data that we have not presented suggest that health districts within the Trent region vary considerably in the neonatal intensive care workload which they generate. At present throughout the Trent region comparatively little resource is allocated to very immature infantsfor example, during the year of the study approximately $2 \cdot 3 \%$ of neonatal admissions (106 infants) were of babies of 26 weeks' gestation or less and $3 \cdot 3 \%$ of admissions (149 infants) were of babies weighing $1000 \mathrm{~g}$ or less. In contrast, for two large centres outside the Trent region infants under $1000 \mathrm{~g}$ accounted for between $14 \%$ and $13 \cdot 4 \%$ of all admissions (H Gamsu, $M$ Chiswick, personal communications).

We believe that the situation in the Trent region reflects the problems of neonatal care throughout the United Kingdom - that is, a service coping with demand by working above and beyond the funded capacity and consequently jeopardising quality of care. If the service is to progress the problem of the gap between demand and provision must be resolved.

We thank all the medical, nursing, and administrative staff of the 17 Trent perinatal units, without whose help this work would not have been possible. Professor M Clarke provided great help with the manuscript. This work was funded by the Trent Regional Health Authority. PB was funded as an MRC training fellow.

\section{Appendix}

British Paediatric Association and British Association for Perinatal Paediatrics definition of intensive care (levels 1 and 2) and special care

\section{INTENSIVE CARE LEVEL 1}

(1) Babies receiving assisted ventilation (intermittent positive pressure ventilation, intermittent mandatory ventilation, continuous positive airway pressure) and in the first 24 hours after withdrawal of assisted ventilation

(2) Babies receiving total parenteral nutrition.

\section{INTENSIVE CARE LEVEL 2}

(1) Babies with cardiorespiratory disease which is unstable, including recurrent apnoea requiring constant attention

(2) Babies who have had major surgery, particularly those in the first 24 hours after such surgery

(3) Babies of less than 30 weeks' gestation during the first 48 hours after birth

(4) Babies who are having convulsions

(5) Babies transported by the staff of the unit concerned. This would usually be between hospitals for special investigations or treatment

(6) Babies having major medical procedures, such as arterial catheterisation, peritoneal dialysis, or exchange transfusion.

SPECIAL CARE

(1) Babies who require continuous monitoring of respiration or heart rate or by transcutaneous transducers

(2) Babies who are receiving additional oxygen 
(3) Babies who are receiving intravenous glucose and electrolyte solutions

(4) Babies who are being tube fed

(5) Babies who have had minor surgery in the previous 24 hours

(6) Babies with a tracheostomy

(7) Dying babies

(8) Babies who are being barrier nursed

(9) Babies who are receiving phototherapy

(10) Babies who are receiving special monitoring (for example, frequent glucose or bilirubin estimations)

(11) Other babies receiving constant supervision (for example, babies whose mothers are drug addicts)

(12) Babies receiving antibiotics

(13) Babies with conditions requiring radiological examination or other methods of imaging.

1 Royal College of Physicians of London. Medical care of the newborn in England and Wales. London: Royal College of Physicians of London, 1988.

2 Anonymous. Medical care of newborn babies [Editorial]. Lancet 1988:ii: 1344-6.

3 Department of Health and Social Security Expert Group on Special Care for
Babies. Report. London: DHSS, 1971. Chairman Sir Wilfrid Sheldon.) Report on Public Health and Medical Subjects, No 127

4 British Paediatric Association and Royal College of Obstetricians and (jynaecologists Liaison Committee. Recommendations for the improvement of infant care during the perinatal period in the l'nted Kingdom. A discussion document. London: British Paediatric Association and Royal College of Obstetricians and Gynaecologists, 1977

5 British Association of Perinatal Medicine Working Group. Referrals for neonatal medical care in the United Kingdom over one year. $\mathrm{Br} M \mathrm{Md}$ 1989;298: 169-72.

6 British Paediatric Association and British Association for Perinatal Paediatrics. Calegories of babies requiring neonatal care. London: British Paediatric Association and British Association for Perinatal Paediatrics, 1984.

7 McCullagh P, Nelder J. Models for data with a constant coefficient of variation. In: Generalised linear models. London: Chapman and Hall, variation. In:

8 Payne CD. The generalised linear interactive modelling system. Oxford: Numerical Algorithms Group, 1985

9 Simpson H, Walker G. Estimating the cots required for neonatal intensive care. Arch Dis Child 1981;56:90-3.

10 South East Thames Regional Perinatal Monitoring Group. Neonutal intensive care services. A draft strategy 1985-1995. London: South East Thames Regional Health Authority, 1987

11 Speidel B. Skimping on care of the newborn is false economy. Br Med $\mathcal{f}$ 1986;293:575

(Accepted 5 October 1989)

\title{
Needle aspiration of amoebic liver abscess
}

\author{
M P Sharma, R R Rai, S K Acharya, J C Samant Ray, B N Tandon
}

\section{Abstract}

Objective-To determine the value of needle aspiration in uncomplicated amoebic liver abscess.

Design-Randomised case-control study with a minimum follow up of one year, comparing patients treated with drugs alone with those treated with additional needle aspiration.

Setting-Referral based gastroenterology clinic.

Patients - 39 Consecutive patients with amoebic liver abscess in the right lobe, of whom 37 completed the study.

Intervention-Metronidazole $2.4 \mathrm{~g} /$ day was given to all patients for $\mathbf{1 0}$ days. Needle aspiration of the abscess was performed in 19 patients on the day of admission to hospital.

Main outcome measures-Abdominal pain, fever, anorexia, and hepatomegaly were measured. Erythrocyte sedimentation rate, serum aspartate, and alanine aminotransferase activities, and alkaline phosphatase activity were also measured.

Results-Clinical improvement was similar in both groups of patients. Improvement in haematological and biochemical variables and rates of healing of cavities were also similar.

Conclusions-Chemotherapy with potent tissue amoebicidal drugs such as metronidazole is optimally effective in treating amoebic liver abscess, and in uncomplicated cases routine aspiration is not required.

\section{Department of}

Gastroenterology and

Microbiology, All India

Institute of Medical

Sciences, New Delhi-

110029 , India

M P Sharma, DM, associate professor

R R Rai, DM, senior resident

S K Acharya, DM, assistant professor

J C Samant Ray, MD,

assistant professor

B N Tandon, MD, professor

Correspondence to:

Dr Sharma. nidazole treatment has also been reported, emphasising the importance of aspiration. ${ }^{3}$ Only few controlled trials of needle aspiration of amoebic liver abscess have been carried out. We compared the immediate and late effects of metronidazole treatment alone with a regimen comprising needle aspiration and metronidazole.

\section{Patients and methods}

Thirty nine patients with amoebic liver abscess diagnosed by using the standard accepted criteria ${ }^{4}$ entered the study. All patients had positive serological results for Entamoeba histolytica (1:400 or more by enzyme linked immunosorbent assay (ELISA) and indirect haemagglutination) and one or more lesions occupying space in the right lobe of the liver with characteristic features of amoebic liver abscess on ultrasound examination. ${ }^{5}$ Patients with an abscess in the left lobe, multiple liver abscesses, and impending rupture were excluded from the study. The patients were admitted to the Rajgarhia liver unit of this institute during January 1985 to October 1987 and were randomised to receive drug treatment alone (non-aspiration group) $(n=20)$ or drug treatment and aspiration $(n=19)$. All patients were treated with metronidazole $2.4 \mathrm{~g} /$ day in divided doses for 10 days. Aspiration of the abscess was carried out under aseptic conditions on the day of admission to hospital, and a mean of $682 \mathrm{ml}$ (range $210-1500 \mathrm{ml}$ ) pus was drained.

All patients were subjected to a thorough clinical examination. Four clinical variables-abdominal pain, fever, anorexia, and hepatomegaly - were assessed on the first, fourth, and 10th day. A reduction in pain by $75 \%$, improvement in appetite by $50 \%$, no fever for at least 72 hours, and regression of hepatomegaly by $30 \%$ or more were considered as definite criteria of improvement and successful treatment. Subsequently the patients were evaluated every three months for a minimum of 12 months. Haematological (erythrocyte sedimentation rate and total and differential counts) and biochemical studies (serum aspartate and alanine aminotransferase activities, and alkaline phosphatase activity) were carried out in all patients on the first, fourth, and 10th day. A raised erythrocyte sedimentation rate of $>30 \mathrm{~mm}$ in the first hour, total leucocyte count of $12.0 \times 10^{4} / 1$, serum alkaline phosphatase activity of 13 King-Armstrong units and aspartate aminotransferase activity $>40 \mathrm{U} / \mathrm{l}$ were considered 